\title{
Nondiffracting Accelerating Wave Packets of Maxwell's Equations
}

\author{
Ido Kaminer, Rivka Bekenstein, Jonathan Nemirovsky, and Mordechai Segev \\ Physics Department and Solid State Institute, Technion, Haifa 32000, Israel
}

(Received 3 February 2012; published 16 April 2012)

\begin{abstract}
We present the nondiffracting spatially accelerating solutions of the Maxwell equations. Such beams accelerate in a circular trajectory, thus generalizing the concept of Airy beams to the full domain of the wave equation. For both TE and TM polarizations, the beams exhibit shape-preserving bending which can have subwavelength features, and the Poynting vector of the main lobe displays a turn of more than $90^{\circ}$. We show that these accelerating beams are self-healing, analyze their properties, and find the new class of accelerating breathers: self-bending beams of periodically oscillating shapes. Finally, we emphasize that in their scalar form, these beams are the exact solutions for nondispersive accelerating wave packets of the most common wave equation describing time-harmonic waves. As such, this work has profound implications to many linear wave systems in nature, ranging from acoustic and elastic waves to surface waves in fluids and membranes.
\end{abstract}

PACS numbers: 42.25.- p, 03.50.De, 41.20.Jb, 41.85.-p

The research on accelerating beams has been growing rapidly since it was brought into the domain of optics in 2007 [1]. An ideal paraxial accelerating beam is propagating along a parabolic trajectory, while preserving its amplitude structure indefinitely, being a nondiffracting wave packet. The effect is caused by interference: the waves emitted from all points on the Airy beam profile maintain a propagation-invariant Airy structure, which shifts laterally along a parabola. This beautiful phenomenon has led to many intriguing ideas ranging from guiding particles along a curve [2], and generating self-bending plasma channels [3] to recent studies on shape-preserving accelerating beams in nonlinear optics [4-7]. In addition, it is possible to find beams accelerating along arbitrary curves at the expense of these beams being not shape-preserving [8]. All of these beams are solutions of the paraxial wave equation, where the beam trajectory is fundamentally limited to small (paraxial) angles, and when it bends to larger angles-the beam is no longer shape preserving. As a result, the transverse acceleration of Airy beams is always restricted to small angles. This restriction is a serious limitation, because spatial acceleration means that the propagation angle continuously increases, and eventually, after physically relevant distances, the beam trajectory inevitably reaches a steep angle, and the beam dynamics as a whole always goes into the nonparaxial regime. That is, a paraxial accelerating beam is moving along a curve which bends ever faster, and eventually it is bound to break its own domain of existence. Several attempts to find an accelerating beam beyond the paraxial regime have shown a complete breakup: part of the beam becomes evanescent while the other part quickly deforms while exhibiting only very small trajectory bending [9]. Most notable is a recent pioneering work [10] where the caustics method is stretched from (paraxial) ray optics to the nonparaxial regime, predicting beams that bend to large angles.
However, as we discuss below, the caustics method cannot provide shape-preserving (nondiffracting) solutions. The recent interest in nonparaxial accelerating beams brings about a series of fundamental questions: Can a beam bend itself to large nonparaxial angles? If it does, would such a nonparaxial accelerating beam be nondiffracting (shape preserving) as it is in the paraxial limit? Such a beam should not be restricted by any physical parameters, and should be able to bend from a launch angle of zero all the way to angles close to $90^{\circ}$ - perpendicular to the original direction of propagation. The dynamics of light is governed by the Maxwell equations; are there any accelerating nondiffracting solutions to the Maxwell equations?

Here, we present nonparaxial spatially accelerating shape-preserving beams. These accelerating beams are the complete set of general solutions to the full Maxwell equations, for any monochromatic fields. These nonparaxial accelerating beams propagate along a circular trajectory, therefore asymptotically reaching $90^{\circ}$ angles, completing a quarter of a circle, after which diffraction broadening takes over and the beams spread out. As a validity test, we prove that taking these beams to the paraxial limit recovers the known paraxial Airy beams. Thus, an accelerating beam of electromagnetic waves (a wave packet satisfying Maxwell's equations) is the beam we present here, and that the Airy beam found in [1] is actually our solution taken to the paraxial limit. We find the solutions for both TE and TM, thereby generalizing to arbitrary polarization. Importantly, what we present here is a new class of nondiffracting solutions to the Helmholtz equation: solutions that selfbend, unlike all previously known nondiffracting solutions of Helmholtz equations (Bessel beams) that propagate on straight trajectory $[11,12]$. Generally, the beams we find exhibit shape-preserving bending with subwavelength features, and the Poynting vector of their main lobe displays a turn of more than $90^{\circ}$. We show that these accelerating 
beams are self-healing, and analyze their properties when they are emitted from finite apertures. Additionally, we show that any given circular trajectory can support an entire family of accelerating solutions, whereby their superpositions form periodic accelerating beams. Finally, the fact that our self-bending beams are solutions to the full wave equation makes this work applicable beyond optics, practically for any time-harmonic wave obeying the simple (Helmholtz-type) wave equation: from sound waves to acoustics, surface waves in fluids, and more.

We begin from Maxwell's equations in vacuum, for a TE-polarized electric field $\bar{E}=E_{Y}(x, z, t) \hat{y}$, obeying the Helmholtz equation

$$
\frac{\partial^{2} E}{\partial x^{2}}+\frac{\partial^{2} E}{\partial z^{2}}-\frac{1}{c^{2}} \frac{\partial^{2} E}{\partial t^{2}}=0 .
$$

Equation (1) has full symmetry between the $x$ and $z$ coordinates. Hence, it is logical to seek a shape-preserving beam whose trajectory resides on a circle. As the initial condition, we use $E(x, z=0, t)$ and let the beam propagate in the forward $+z$ direction. Of course, such a beam cannot turn back to propagate in the $-z$ direction; hence, the largest bending expected is a trajectory parallel to the $x$ direction. That is, the beam will asymptotically complete circular motion on a quarter of a circle. To seek such motion which is also shape-invariant (diffraction-free), it is convenient to transform Eq. (1) to the rest frame of the beam. Since the motion is on a circle, we transform to polar coordinates $r, \theta$, by taking $z=r \sin (\theta), x=r \cos (\theta)$, and seek shape-preserving solutions of the form $E=$ $U(r) e^{i \alpha \theta-i \omega t}$, where $\alpha$ is some real number, and $\omega$ is the temporal frequency. The result is a monochromatic beam, which is shape preserving along any circular curve. The radial function $U(r)$ must satisfy:

$$
r \frac{d}{d r}\left(r \frac{d U}{d r}\right)+\left[-a^{2}+\left(\frac{\omega^{2}}{c^{2}}\right) r^{2}\right] U=0 .
$$

The exact solutions of Eq. (2) are the Bessel functions $U=J_{\alpha}[(\omega / c) r]$. [Actually, there is an additional family of solutions, also from the Bessel family, but those diverge at the origin; hence, we will not discuss them here.] A related method was used recently by Hacyan [13], to find electromagnetic waves that accelerate relativistically in time.

To unravel the physics of our solution, it must be transformed back to the coordinates $x, z$, and separated into forward and backward propagating waves. We do it through the Fourier transform of the beam, which is confined to reside on a circle of radius $k=\omega / c=2 \pi / \lambda$ in the $k_{x}-k_{z}$ plane (see the diagram in the center of Fig. 1). The top half of the diagram (positive $k_{z}$ ) gives the forward propagating part of the beam, while the bottom half (negative $k_{z}$ ) gives the backward propagating part. The forward propagating part is the actual accelerating beam from a single source at $z=0$. Importantly, this beam does not have the Bessel structure, but only asymptotically half of it

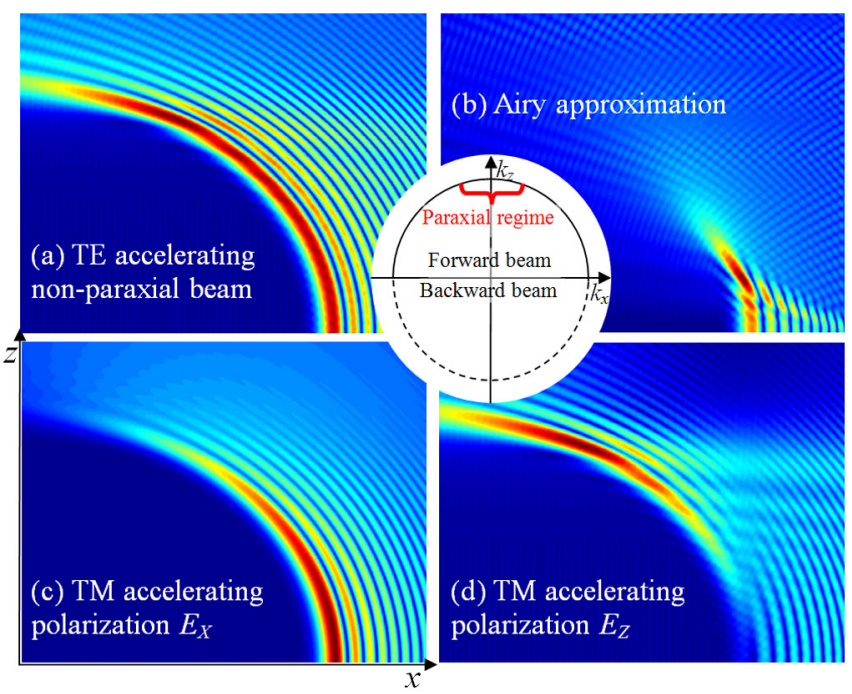

FIG. 1 (color online). Accelerating beams of the Maxwell equations. (a) The forward-accelerating beam of TE polarization ( $\alpha=150$ ) reaches a trajectory which is almost vertical, while exhibiting nondiffracting (shape-preserving) acceleration. (b) The paraxial approximation yields an Airy beam which accelerates only for a short distance before breaking up. (c), (d) The forward propagating beam of TM polarization ( $\alpha=150$ ). The power transfers from the $x$ polarization (c) to the $z$ polarization (d). All figures are simulated with $\lambda=1 \mu \mathrm{m}$ and in a square of $35 \mu \mathrm{m} \times 35 \mu \mathrm{m}$. The diagram in the center shows the Fourier plane where the beam is confined in a circle describing the propagating plane waves. The top (bottom) half stands for the forward (backward) beam.

[14]. This can be calculated by integration over the top half of the circle (angles 0 to $\pi$ ), as sketched on the top half of the diagram on Fig. 1.

$E_{Y}^{+}(x, z)=\int_{0}^{\pi} e^{i \alpha k_{\theta}} e^{i k\left[x \cos \left(k_{\theta}\right)+z \sin \left(k_{\theta}\right)\right]} d k_{\theta} \equiv J_{\alpha}^{+}(k x, k z)$.

Where $J_{\alpha}^{+}$is "half a Bessel" (because integrating the expression from $-\pi$ to $\pi$ yields the $\alpha$-order Bessel function $J_{\alpha}(k r)$ ). Here, $\alpha$ can be any real number (not necessarily an integer) since we are not restricted to periodic boundary conditions (as the beam never completes a full circle). Figure 1(a) shows the accelerating solution of Eq. (3), for $\lambda=1 \mu \mathrm{m}$ and $\alpha=150$. It is important to emphasize that the axes have the same scale in all figures in this Letter, unlike the usual representation of Airy beams, as appears in many papers, where the curvatures are usually exaggerated to highlight the beam bending, by making use of unequal scales. Figure 1(a) shows that the beam is indeed nondiffracting (shape preserving), but only up to an angle close to $90^{\circ}$. We should discuss the reason for this limit: Mathematically, it is clear that a Bessel solution is exact and shape preserving. However, the physical beam, generated by the initial condition at plane $z=0$, is only "half a Bessel". In what sense is this beam nondiffracting? When $\alpha>0$, the exact Bessel beam is antisymmetric at 
$z=0$ with respect to the origin: two main lobes positioned at opposite sides of $x=0$ and their oscillating tails stretch toward plus and minus infinity on their right and left sides, respectively. But, the phase of the beam is what makes the beam antisymmetric: it follows from the anticlockwise rotation of the beam. This rotation makes the right half of the beam propagate forward and to the left [Fig. 1(a)], while the left half propagates backward and to the right. The latter contradicts the physical boundary conditions. When cutting the backward propagating waves in Fourier space, we are left with an almost-exact Bessel shape confined on the right side of the $x$ axis. Only when the bending gets close to $90^{\circ}$, the nondiffracting property breaks, where the two "half Bessel wave packets" were supposed to meet and interfere ([14]). Mathematically, larger $\alpha$ (larger angular momentum) gives a better separation, hence also a more accurate nondiffracting propagation.

The solution for the TM polarization is found through a similar procedure for the magnetic field, and from it the TM electric field components are found to be

$E_{Z}^{+}(x, z)=\frac{1}{2} J_{\alpha+1}^{+}(k x, k z)+\frac{1}{2} J_{\alpha-1}^{+}(k x, k z)$,

$E_{X}^{+}(x, z)=\frac{i}{2} J_{\alpha+1}^{+}(k x, k z)-\frac{i}{2} J_{\alpha-1}^{+}(k x, k z)$.

This TM solution is of special interest: each of its polarization components is not shape preserving on its own, as shown in Figs. 1(c) and 1(d), but the total intensity of the TM beam does preserve its shape. That is, as the beam bends by $90^{\circ}$, the power is transferred from the $x$ component to the $z$ component of the field. This shows that the accelerating beam not only bends but actually rotates, similar to the phase front of the beam which also rotates by $90^{\circ}$, staying normal to the beam trajectory at all times. A natural extension comes from the superposition of the TE and TM beams, which yields a vectorial solution of general polarization.

Generalizing Eq. (3) to arbitrary polarization gives the full family of vectorial 3D accelerating beams. We still choose the trajectory of acceleration in the $x z$ plane, but allow a plane wave in $y$. This leaves three functions in $k$ space that relate to the electric field via Eqs. (5) [similar to Eq. (3)]

$$
\begin{aligned}
E_{j}^{+}(x, y, z) & =e^{i k_{y} y} \int_{0}^{\pi} f_{j}\left(k_{\theta}\right) e^{i k_{x z}\left[x \cos \left(k_{\theta}\right)+z \sin \left(k_{\theta}\right)\right]} d k_{\theta}, \\
j & =x, y, z,
\end{aligned}
$$

where $k_{x z}, \quad k_{y}$ must satisfy the conditions: $k_{x z} \cos \left(k_{\theta}\right) f_{x}\left(k_{\theta}\right)+k_{y} f_{y}\left(k_{\theta}\right)+k_{x z} \sin \left(k_{\theta}\right) f_{z}\left(k_{\theta}\right)=0$, and $k_{x z}^{2}+k_{y}^{2}=k^{2}$. Each polarization is therefore composed of a superposition of solutions of Eq. (3) in the TE and the TM polarization, multiplied by a plane wave which only changes the effective wave number from $k$ to $k_{x z}$. Superpositions of fields with different $k_{y}$ should give beams which are confined in the $y$ direction, extending the solutions to 3D.

To highlight the impressive angle of bending, we note that it is actually possible to double the angle, by launching the beam at an angle opposite to the direction of bending. See Fig. 2(a) for an example of a beam that is launched at an angle of $-65^{\circ}$ and subsequently bends all the way to $65^{\circ}$, completing a turn of $130^{\circ}$. In theory, the maximal bending is limited to $180^{\circ}$ asymptotically because the boundary conditions allow only forward propagating waves. In practice, we measure the bending in Fig. 2(a) by the difference between the Poynting vectors of the main lobe at the incoming plane and at the outgoing plane. One can prove that the Poynting vector of the TM polarization is exactly the same.

Having found the accelerating solutions of Maxwell's equations, it is interesting to examine the small angles limit of the expression in Eq. (3), and see if it recovers the paraxial Airy solution. To do that, we recall a property of the Bessel function stating that the maximum of the main lobe occurs close to $x=\alpha / k$. Thus, to make the approximation in the correct range, we take $x=\alpha / k+\Delta x$ and assume $\Delta x$ and $z$ to be small. We also assume $\alpha$ to be very large, so that the exponent oscillates very fast and cancels out most of the contribution of the nonparaxial regime. In the limit of large $\alpha$, we expand the cosine and sine by a Taylor series around $\pi / 2$, up to third order. The result is an integral that is solved analytically to yield

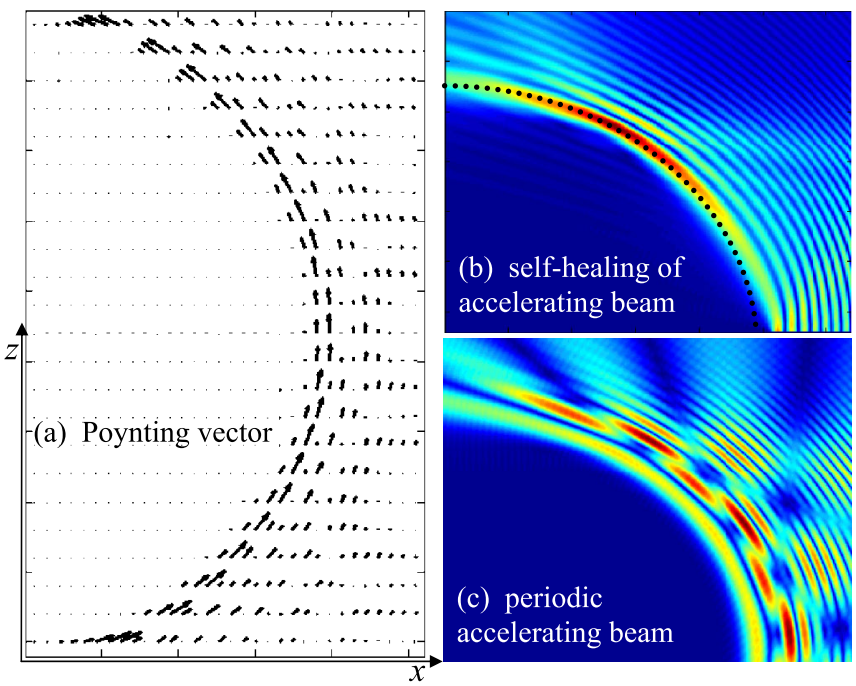

FIG. 2 (color online). Properties of the Bessel-like accelerating beams. (a) The Poynting vector of the TE/TM polarization, when launched with an initial angle and bends by $130^{\circ}$. (b) The self-healing property "reviving" the first lobe that was initially cut out. The dashed black curve describes the original trajectory of the first lobe of a perfect accelerating beam. (c) A periodic accelerating beam composed of two jointly accelerating components $\alpha=150$ and $\alpha=165$ with equal coefficients. All figures are simulated with $\alpha=150, \lambda=1 \mu \mathrm{m}$ and in a square of $30 \mu \mathrm{m} \times 45 \mu \mathrm{m}$ (a) or $35 \mu \mathrm{m} \times 35 \mu \mathrm{m}$ (b), (c). 


$$
\begin{aligned}
E_{Y}^{+}(x, z) \propto & A i\left(-2^{1 / 3} \alpha^{-1 / 3} k \Delta x-2^{-2 / 3} \alpha^{-4 / 3} k^{2} z^{2}\right) \\
& \times e^{i k z+i k z \Delta x \alpha^{-1}-(i / 3) k^{3} z^{3} \alpha^{-2}},
\end{aligned}
$$

where $A i$ is the Airy function. Note the $z^{3}$ term characteristic of the paraxial Airy beam, indicating acceleration at a parabolic trajectory A direct consequence is that there is a unique relation between the parameter $\alpha$ and the acceleration (from the trajectory $\Delta x=-g z^{2} / 2$ ), which is simply $g=k / \alpha$. Hence, the acceleration $g$ is smaller for larger $\alpha$, which makes sense because higher orders of $\alpha$ give circular motion with larger radii, so the radial acceleration is indeed smaller. Another conclusion is that if we try to approximate an accelerating beam which is a superposition of several $\alpha$ 's, we get a superposition of Airy beams with different accelerations. This is why the paraxial accelerating beam must be a single Airy function, with a uniquely defined acceleration, whereas the nonparaxial accelerating beams can support a family of beams of different shape (different $\alpha$ 's) which all accelerate at the same trajectory. Finally, for small values of $\alpha$, we find accelerating beams that cannot exist in the paraxial regime at all. Those beams are mainly made up of very high spatial frequencies; hence, trying to construct an Airy beam with these spatial frequency constituents gives rise to a beam that breaks up after a very short propagation distance. See Fig. 1(b) for an example, with $\alpha=150$, where the parabolic acceleration survives for a very short distance only.

It is now worth while to compare the known features of the Airy beam to our new nonparaxial accelerating beam. To this end, we notice that the self-healing effect [15] also exists in our nonparaxial beams: see Fig. 2(b) for an example, where the main lobe is initially cut out (blocked). The second lobe gets more power and its trajectory bends more-to replace the first lobe; see the dotted black line on Fig. 2(b) marking the trajectory of the original first lobe. When this "replacement" occurs, each lobe is shifted by a steeper bending to replace the lobe on its left.

Another property common for both the Airy beam and the nonparaxial accelerating beam is that both are not square integrable; hence, they carry infinite power. In this context, launching either of them from a finite aperture yields a beam that accelerates over a finite propagation range only. As with the Airy beam, a longer tail in the nonparaxial case allows for more lobes to exhibit shapepreserving propagation for larger distances. Interestingly, the reason that the nonparaxial accelerating beam carries infinite power comes from only two singular points in $k$ space, which are placed at the edges $(-k$ and $k$ ) bordering the regime of evanescent waves. Removing these singular points leaves an accelerating beam of finite power, since then the $k$-space spectrum becomes square integrable (unlike the paraxial spectrum of the Airy which is unbounded). Physically, some part of the spatial spectrum will always be removed, since the edges of the $k$ space represent waves moving transversely perpendicular to $z$. At the same time, a rather short tail (of about twice the radius of the trajectory) is sufficient to make the first lobes bend into a deep nonparaxial angle (more than $50^{\circ}$ ). Consequently, a nonparaxial accelerating beam launched from a finite aperture (thus carrying finite power) will bend on a circular curve while maintaining a virtually propagation-invariant shape for the majority of the physically accessible one quarter of a circle.

Finally, another difference between the Airy beam and the nonparaxial accelerating beam is that, unlike the Airy beam, the Bessel-like nonparaxial accelerating beams cannot be simply scaled (by squeezing or stretching the $x$ axis) to control the acceleration curve. Rather, in the nonparaxial case different values of $\alpha$ imply different orders of the Bessel-like function, which affects the widths of the lobes indirectly.

When coming to examine the nonparaxial accelerating solutions of Eqs. (3) and (4), we note that any superposition of these solutions, with different values of $\alpha$, also gives an accelerating beam which is propagating on the same curved trajectory. Such superposition accelerates in unison, but it is not shape preserving: it is a breather, with a periodicity depending on the difference between the values of $\alpha$. Thus, an infinite family of periodic accelerating beams can be generated from superpositions. Figure 2(c) displays such a periodic accelerating beam. Note that some of the periodic accelerating beams have finite power, due to the destructive interference at the tails. Mathematically, this happens when the singular points in $k$ space $\left(k_{\theta}=0, \pi\right)$ are canceled by summing two or more waves, as in the case of the $x$ component of the TM polarization (which is also a legitimate periodic solution for TE). Many other examples of finite power periodic accelerating beams can be generated from Eq. (5) for mixed polarizations.

Before closing, we note that accelerating beams can also be found through methods relying on caustics $[8,10,16]$. A recent paper [10] proposed using the caustic method to generate nonparaxial accelerating beams. This method is based on ray-optic principles but taken into nonparaxial angles. This way, accelerating beams moving along an arbitrary curve can reach large bending angles. However, while this method constrains the main lobe to accelerate along the predesigned curve, it does not determine how the rest of the beam is propagating. In practice, the beam is considered accelerating, but it is not nondiffracting: after some distance, diffraction effects smear the beam structure and acceleration stops [8,10]. Hence, such "causticdesigned accelerating beams" are different from the paraxial Airy beams, from nonlinear accelerating beams [4-7], and from the nonparaxial accelerating beams described here, which are all nondiffracting: for all of these, the entire beam is accelerating with a propagation-invariant amplitude, whereas caustic-designed accelerating beams are not meant to be propagation invariant. 
To summarize, we have found nonparaxial accelerating beams and nonparaxial periodically oscillating accelerating beams. These beams are the full vector solutions of Maxwell's equation for shape-preserving accelerating beams. Moreover, in their scalar form, these beams are the exact solutions for nondispersive accelerating wave packets of the simple and most common wave equation describing time-harmonic waves. As such, the work presented in this Letter has profound implications to almost any linear wave system in nature, ranging from sound waves and surface waves in fluids to many kinds of classical waves. In this spirit, it is now clear that the phenomenon of accelerating waves is not the result of a specific unusual behavior of the Schrödinger equation (which is equivalent to the paraxial wave equation), as one may think from reading the first paper pioneering this subject [17]. In a similar vein, this work shows that nonparaxial nondiffracting beams are no longer necessarily Bessel-like beams [11,12], which always propagate on a straight line, but now include also self-bending beams. To complete the picture, future work should study the possibility of 3D accelerating beams, including those with trajectories that do not lie in a single plane. In practical terms, this work brings accelerating beam optics into the subwavelength regime, through the less-than-wavelength features of our solutions, facilitating higher resolution for particle manipulation.

This work was supported by an Advanced Grant from the European Research Council, by the Israel Science Foundation, and by the USA-Israel Binational Science Foundation.

Note added in proof.-It has been brought to our attention that nonparaxial accelerating beams have recently been demonstrated (see [18]), designed using a causticbased approach. Fundamentally, caustic-based methods cannot be used to find the exact accelerating nondiffracting solutions to the Helmholtz equation, as described here. However, the authors of [18] used an approximate diffraction integral analysis to seek beams with a width-invariant caustic, and found that such beams have a circular trajectory. Certainly, one can view the experiments in [18] as the first observation of the accelerating shape-preserving nonparaxial beams we predicted in our Letter here.
[1] G. A. Siviloglou and D. N. Christodoulides, Opt. Lett. 32, 979 (2007); G. A. Siviloglou, J. Broky, A. Dogariu, and D. N. Christodoulides, Phys. Rev. Lett. 99, 213901 (2007).

[2] J. Baumgartl, M. Mazilu, and K. Dholakia, Nature Photon. 2, 675 (2008).

[3] P. Polynkin, M. Kolesik, J. V. Moloney, G. A. Siviloglou, and D.N. Christodoulides, Science 324, 229 (2009).

[4] I. Kaminer, M. Segev, and D. N. Christodoulides, Phys. Rev. Lett. 106, 213903 (2011);

[5] A. Lotti, D. Faccio, A. Couairo, D. G. Papazoglou, P. Panagiotopoulos, D. Abdollahpour, and S. Tzortzakis, Phys. Rev. A 84, 021807 (2011).

[6] I. Dolev, I. Kaminer, A. Shapira, M. Segev, and A. Arie, Phys. Rev. Lett. 108, 113903 (2012).

[7] R. Bekenstein and M. Segev, Opt. Express 19, 23706 (2011).

[8] E. Greenfield, M. Segev, W. Walasik, and O. Raz, Phys. Rev. Lett. 106, 213902 (2011).

[9] A. V. Novitsky and D. V. Novitsky, Opt. Lett. 34, 3430 (2009); L. Carretero, P. Acebal, S. Blaya, C. García, A. Fimia, R. Madrigal, and A. Murciano, Opt. Express 17, 22432 (2009).

[10] L. Froehly, F. Courvoisier, A. Mathis, M. Jacquot, L. Furfaro, R. Giust, P. A. Lacourt, and J. M. Dudley, Opt. Express 19, 16455 (2011).

[11] J. Durnin, J. J. Miceli, Jr., and J. H. Eberly, Phys. Rev. Lett. 58, 1499 (1987).

[12] J.A. Stratton, Electromagnetic Theory (Classic Reissue, IEEE Press, New Jersey, 2007).

[13] S. Hacyan, J. Opt. 13, 105710 (2011).

[14] Similar to a temporally accelerating pulse which is also split in two halves, corresponding to positive and negative group velocities; see I. Kaminer, Y. Lumer, M. Segev, and D. N. Christodoulides, Opt. Express 19, 23132 (2011).

[15] J. Broky, G. A. Siviloglou, A. Dogariu, and D. N. Christodoulides, Opt. Express 16, 12880 (2008).

[16] Y. Kaganovsky and E. Heyman, Opt. Express 18, 8440 (2010).

[17] M. V. Berry and N.L. Balazs, Am. J. Phys. 47, 264 (1979).

[18] F. Courvoisier, A. Mathis, L. Froehly, R. Giust, L. Furfaro, P.-A. Lacourt, M. Jacquot, and J. M. Dudley, arXiv:1202.3318v1. 